\title{
Propagation of Uncertainty in the MUSIC Algorithm Using Polynomial Chaos Expansions
}

\author{
Thomas Van der Vorst $^{1}$, Mathieu Van Eeckhaute ${ }^{1}$, Aziz Benlarbi-Delaï ${ }^{2}$, Julien Sarrazin ${ }^{2}$, François Horlin ${ }^{1}$, \\ Philippe De Doncker ${ }^{1}$ \\ ${ }^{1}$ Université libre de Bruxelles (ULB), Av. Roosevelt 50, 1050 Brussels, Belgium \\ 2 Sorbonne Universités, UPMC Univ Paris 06, UR2, L2E, F-75005 Paris, France \\ Contact : tvdvorst@ulb.ac.be
}

\begin{abstract}
Polynomial chaos expansions are used to analyze the propagation of uncertainties on array parameters in Angleof-Arrival estimation performed by the MUSIC algorithm.

Index Terms-MUSIC, Polynomial chaos, Propagation of uncertainty, Localization
\end{abstract}

\section{INTRODUCTION}

Among all high resolution methods of Angle-of-Arrival (AoA) estimation, the MUSIC algorithm is certainly one of the most popular [1]. In order to give precise results, the MUSIC algorithm needs thorough knowledge of the array parameters, i.e. the gain and phase of each sensor (antenna and front-end). As this method is implemented on physical arrays of antennas, the array parameters are influenced by different factors, such as temperature and aging, and may fluctuate in time. Therefore, the array parameters are subject to uncertainties. We propose to estimate the influence that the uncertainties on the array parameters have on the output of MUSIC.

To study propagation of uncertainty, polynomial chaos expansions have already been used in electromagnetics, e.g. in ray-tracing [2], or in dosimetry [3]. From the probability density function (PDF) of the input random variables of a process, the polynomial chaos expansions allow one to determine the statistical moments of the output of the process [4].

In this paper, we aim to relate the variance of the AoA estimated with MUSIC to the PDF of the phase and gain of the sensors in an antenna array.

\section{METHOD}

\section{A. The MUSIC algorithm}

MUSIC is a high resolution method for AoA estimation based on the eigenvector decomposition of the sample data covariance matrix [1]. Consider $M$ sensors measuring each $N$ samples. Then, we can write the sample data as the $M \times N$ matrix $\mathbf{Y}$, of which a column is called a snapshot. Suppose there are $d$ incident signals, which we will consider to be sinusoids. Let $\mathbf{S}$ be the $d \times N$ matrix of the associated incident signals. Now define the perturbation matrix $\mathbf{G}=\operatorname{diag}\left(g_{i} e^{j \phi_{i}}\right)$, where $g_{i}$ and $\phi_{i}$ are the gain and the phase of the sensor $i$, respectively. Introducing the steering matrix $\mathbf{A}$, we can write the sample data matrix as $\mathbf{Y}=\mathbf{G A S}+\mathbf{N}$, where
$\mathbf{N}$ is white noise. The covariance matrix is then obtained : $\mathbf{R}=\mathbf{Y Y}^{H} / N$. This algorithm reduces inherently the noise by averaging the covariance matrix over the samples. Now the idea is to decompose the covariance matrix into eigenvectors. It can be shown [1] that :

$$
\mathbf{R}=\left[\mathbf{U}_{s} \mathbf{U}_{n}\right]\left[\begin{array}{c|c}
\boldsymbol{\Lambda}_{s}+\sigma^{2} \mathbf{I}_{d} & 0 \\
\hline 0 & \sigma^{2} \mathbf{I}_{M-d}
\end{array}\right]\left[\begin{array}{c}
\mathbf{U}_{s}^{H} \\
\mathbf{U}_{n}^{H}
\end{array}\right]
$$

i.e.

$$
\mathbf{R}=\mathbf{U} \boldsymbol{\Lambda} \mathbf{U}^{H}
$$

In this equation, the $\boldsymbol{\Lambda}_{s}$ are the eigenvalues of the correlation matrix, and $\sigma^{2}$ is the noise variance. There are $d$ eigenvalues of the correlation matrix as each one is associated with one signal. The signal eigenvectors $\mathbf{U}_{s}$ span the so-called signal subspace, while the noise eigenvectors $\mathbf{U}_{n}$ span the noise subspace. The signal vectors being obviously orthogonal to the noise subspace, the projection on the noise subspace of a steering vector corresponding to an AoA must be null. Therefore, the inverse of the cost-function $J_{\text {MUSIC }}(\theta)$ shows narrow peaks for directions corresponding to actual AoA, where

$$
J_{\operatorname{MUSIC}}(\theta)=\frac{\left\|\hat{\mathbf{U}}_{n}^{H} \mathbf{a}(\theta)\right\|^{2}}{\|\mathbf{a}(\theta)\|^{2}}=\frac{\mathbf{a}(\theta)^{H} \hat{\mathbf{U}}_{n} \hat{\mathbf{U}}_{n}^{H} \mathbf{a}(\theta)}{\mathbf{a}(\theta)^{H} \mathbf{a}(\theta)}
$$

in which $\mathbf{a}(\theta)$ is the steering vector corresponding to the direction $\theta$.

\section{B. Polynomial chaos expansions}

Let $f$ be the estimated AoA at the output of the MUSIC algorithm. It is a function of the random variables $X_{i}=g_{i}$ or $\phi_{i} . f(X)$ can be expressed as [4]:

$$
f(X)=\sum_{j=0}^{\infty} f_{j} \Psi_{j}\left(\left\{X_{i}\right\}_{i=1}^{2 M}\right)
$$

where the polynomials $\left\{\Psi_{j}\left(\left\{X_{i}\right\}_{i=1}^{2 M}\right)\right\}_{j=1}^{\infty}$ form a polynomial chaos basis of the adequate Hilbert space containing $f$. These multivariate polynomials are products of univariate polynomials, $\Psi_{\boldsymbol{\alpha}}(\mathbf{X}) \equiv \prod_{i=1}^{2 M} \Psi_{\alpha_{i}}^{(i)}\left(X_{i}\right)$. For each input random variable $X_{i}$, univariate polynomials are constructed so that they are orthogonal with respect to the scalar product defined by the PDF of $X_{i}, \varphi_{X_{i}}$ :

$$
\left\langle\Psi_{j}^{(i)}, \Psi_{k}^{(i)}\right\rangle=\int \Psi_{j}^{(i)}(u) \Psi_{k}^{(i)}(u) \varphi_{X_{i}}(u) \mathrm{d} u=\gamma_{j}^{(i)} \delta_{j k}
$$


TABLE I

NUMBER OF SNAPSHOT NEEDED TO AVERAGE OUT NOISE IN THE OUTPUT VARIANCE ESTIMATION

\begin{tabular}{|c|c|c|c|}
\hline SNR [dB] & 20 & 10 & 3 \\
\hline snapshots & 850 & 1000 & 6000 \\
\hline
\end{tabular}

The coefficients $f_{i}$ can be obtained by different methods. We use in this work the projection method, using a GaussLegendre quadrature to evaluate the integrals, as described by Sudret [4]. In practice, the series appearing in (3) is truncated at a certain order $P-1$, which is generally low. The interest of this method is that knowing the PDF of the input variables allows one to deduce statistical information on the output values with a limited number of runs of the MUSIC algorithm. Indeed, the mean and variance of the output are given by:

$$
\begin{gathered}
\bar{f}=\mathrm{E}[f(\mathbf{X})]=f_{0} \\
D_{\mathrm{PC}}=\operatorname{Var}\left[\sum_{j=1}^{P-1} f_{j} \Psi_{j}(\mathbf{X})\right]=\sum_{j=1}^{P-1} f_{j}^{2} \mathrm{E}\left[\Psi_{j}^{2}(\mathbf{X})\right]
\end{gathered}
$$

\section{RESULTS}

\section{A. Implementation}

We consider a linear uniform array (ULA) of $M=4$ sensors, on which a single wave is incident from a given angle (AoA). The sensors are supposed to be subject to random phase and/or gain variations. These inputs are chosen to be uniform random variables with unit mean for the gain $g_{i}$ and zero mean for the phase $\phi_{i}$. Therefore, we expand the output in (3) on a Legendre polynomials series [4]. For a given order of truncation of the series $P-1$, we calculate the coefficients $\left\{f_{i}\right\}_{i=0}^{P-1}$ with a Gauss-Legendre quadrature of order $P$. We must then evaluate the output of the MUSIC algorithm for $P^{2 M}$ particular values of the input vector.

\section{B. Influence of the noise}

The variance of the AoA estimated by MUSIC is caused by two fundamentally different reasons : noise and array parameters uncertainties. To enable the analysis of parameter uncertainty propagation, it is necessary to eliminate the contribution of the noise to the output variance. By averaging over a certain number of snapshots, MUSIC is able to cancel the noise contribution to the variance. In Fig. 1, the output variance of MUSIC is drawn as a function of the number of snapshots, for different SNR values. In this calculation we used a 5th-order Legendre polynomial expansion of the output AoA, a phase interval halfwidth of $25^{\circ}$, and a gain interval halfwidth of 0.5 . The number of snapshots needed to average out the noise, inferred from this figure are given in Table I.

\section{Phase Uncertainty}

To study the dependence of the output variance on the phase uncertainty, we have run the program for only a random phase on one sensor. We set the value of the SNR at $20 \mathrm{~dB}$, and

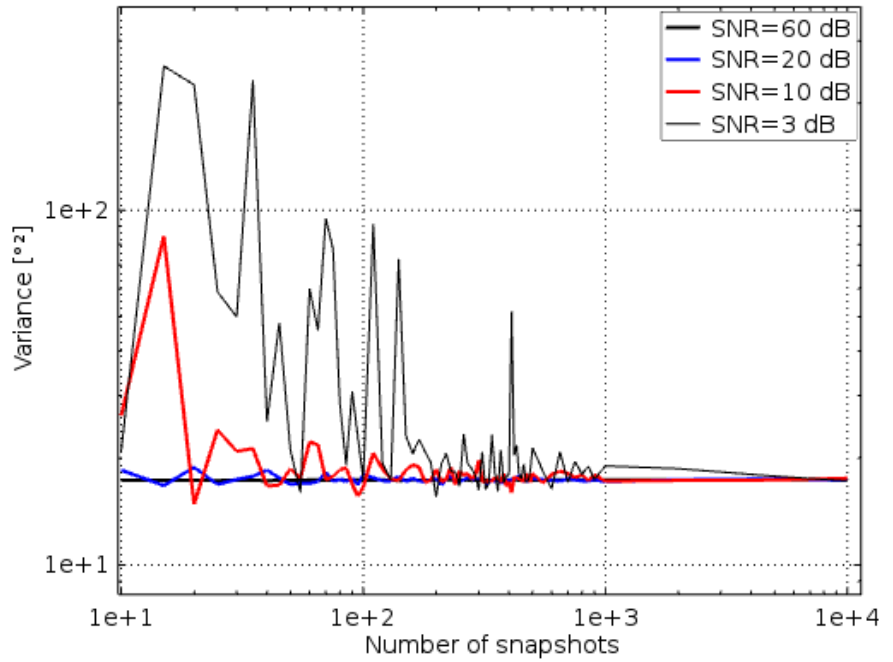

Fig. 1. Variance of the estimated AoA as a function of the number of snapshots used in MUSIC, for different values of SNR

therefore set the number of snapshots at 850 , so that the noise has no contribution to the variance of the estimated AoA. Fig. 2 shows the standard deviation of the output as a function of the input phase interval halfwidth, for different values of the AoA. In this calculation we used a 5th-order Legendre polynomial expansion of the estimated AoA. The first observation is that the standard deviation of the estimated AoA depends linearly of the standard deviation of the input phase. The second important observation is that the output variance grows importantly as the AoA grows.

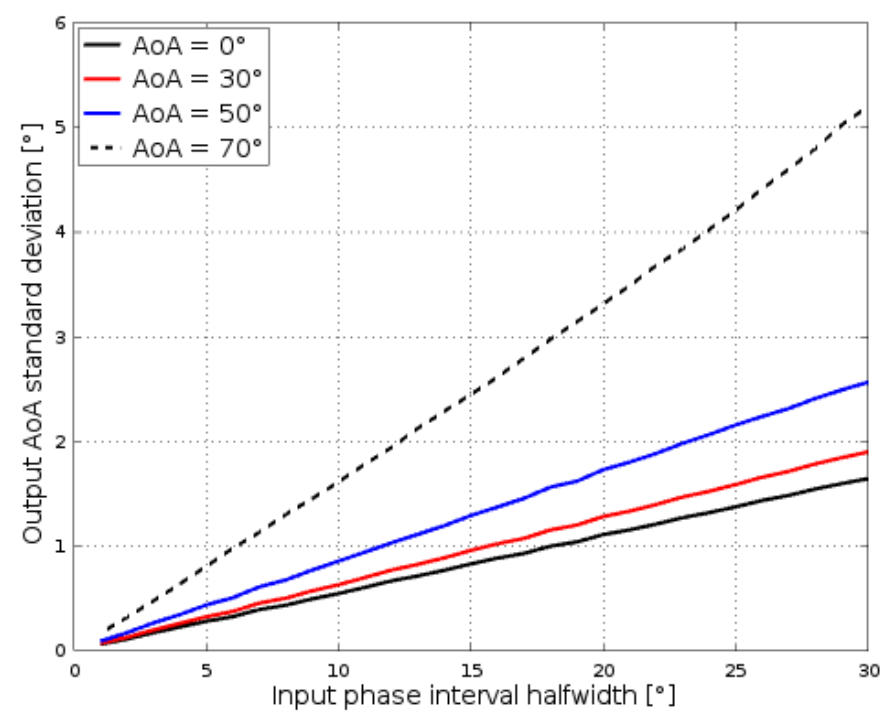

Fig. 2. Standard deviation of the estimated AoA as a function of the input phase interval halfwidth

\section{Gain Uncertainty}

Secondly we extended the calculation to the case of simultaneous gain and phase uncertainties on a single sensor, 
in order to evaluate the influence of the gain uncertainty in the output variance. Fig. 3 shows the normalized dependence of the output standard deviation on the input gain interval halfwidth. The normalization consists in dividing the output standard deviation by the standard deviation obtained without gain uncertainty. The calculation was performed with an AoA of $70^{\circ}$ and a 5th-order Legendre polynomial expansion of the output AoA. We observe that the influence of the gain uncertainty on the output variance is small, but not negligible.

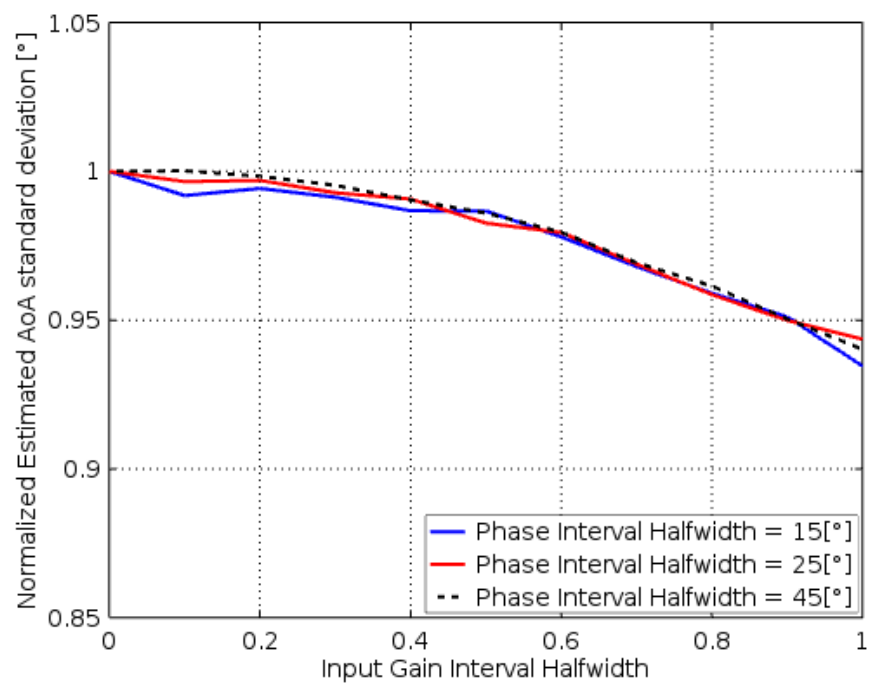

Fig. 3. Normalized standard deviation of the estimated AoA as a function of the input gain interval halfwidth

\section{CONCLUSION}

The number of snapshots needed to average out the noise for estimation of MUSIC output variance has been evaluated. The dependence of the standard deviation of the estimated AoA on the width of the PDF of the phase uncertainty on one sensor was found to be linear, and considerably dependent on the AoA. The contribution of the gain uncertainty to the output variance was found to be small in comparison with the phase uncertainty contribution, but not negligible, and appears for all values of phase uncertainty.

\section{REFERENCES}

[1] R. Schmidt, Multiple Emitter Location and Signal Parameter Estimation, IEEE Transactions on Antennas and Propagation, 34, pp 276-280, 1986.

[2] A. Haarscher, Ph. De Doncker, D. Lautru, Uncertainty propagation and sensitivity analysis in ray-tracing simulations, PIER M, v21, pp. 149-161, 2011.

[3] P. Kersaudy, B. Sudret, N. Varsier, O. Picon, J. Wiart, A new surrogate modeling technique combining Kriging and polynomial chaos expansions - Application to uncertainty analysis in computational dosimetry, Journal of Computational Physics, 286, pp.130-117, 2015.

[4] B. Sudret, Global Sensitivity Analysis Using Polynomial Chaos Expansions, Reliab. Eng. Sys. Saf., 93, pp 964-979, 2008. 